\title{
Patterns of the block approach for structure analysis of chemical elements and issues in materials science
}

\author{
Authors: \\ Boris V. Gusev, \\ Head of the Department of Construction Materials and Technologies at Russian University of Transport, \\ Moscow, Russia, e-mail: info-rae@mail.ru
}

\section{Anatoly A. Speransky,}

Vice President of RAE, Moscow, Russia, e-mail: vibro-vector@yandex.ru

\begin{abstract}
The authors developed a notion of the three-dimensional matrix of chemical elements, which made it possible to eliminate the main disadvantages of tabular forms of the structure of chemical elements developed by D.I. Mendeleev and the international community of IUPAC chemists. The three-dimensional structure is represented as an expanding conic matrix. At the same time, all known chemical elements up to number 118 are combined into four blocks. The block-structure made it possible to substantiate an electron-level formula, even for the proposed new chemical elements of the 5th block E with numbers 119 through 218. This allows for the development of a digital model for calculating interactions of chemical elements and obtaining new types of compounds and materials.
\end{abstract}

Keywords: chemical elements, tabular forms of the periodic structure, three-dimensional matrix, periodicity mechanisms, orbital models of atom electron shells.

For citation: Gusev B.V., Speransky A.A. Patterns of the block approach for structure analysis of chemical elements and issues in materials science. Nanotehnologii v stroitel'stve $=$ Nanotechnologies in Construction. 2019, Vol. 11, no. 1, pp. 76-88. DOI: 10.15828/2075-8545-2019-11-1-76-88.

Machine-readable information on CC-licenses (HTML-code) in metadata of the paper

$<$ a rel="license" href="http://creativecommons.org/licenses/by/4.0/" $><$ img alt="Creative Commons License" style="border-width:0" src="https://i.creativecommons.org///by/4.0/88x31.png" / ></a $><$ br / ><span xmlns:dct="http://purl.org/dc/terms/" href="http://purl.org/dc/dcmitype/Text" property="dct:title" rel="dct:type" $>$ Patterns of the block approach for structure analysis of chemical elements and issues in materials science $</$ span $>$ by $<$ a xmlns:cc $="$ http://creativecommons.org/ns\#" href="Nanotehnologii v stroitel'stve = Nanotechnologies in Construction. 2019, Vol. 11, no. 1, pp. 76-88. DOI: 10.15828/2075-8545-2019-11-1-76-88" property="cc:attributionName" rel="cc:attributionURL" $>$ Gusev B.V., Speransky A.A. $</ a>$ is licensed under a $<$ a rel="license" href="http://creativecommons.org/licenses/ by/4.0/">Creative Commons Attribution 4.0 International License $</ \mathrm{a}>$. $<$ br $/>$ Based on a work at $<$ a xmlns:dct="http://purl.org/dc/terms/" href="http://nanobuild.ru/ en_EN/nanobuild-1-2019/" rel="dct:source">http://nanobuild.ru/en_EN/nanobuild-1-2019/</a $>$.<br />Permissions beyond the scope of this license may be available at <a xmlns:cc="http://creativecommons.org/ns\#" href="info-rae@mail.ru" rel="cc:morePermissions" $>$ info-rae@mail.ru $</ a>$.

The paper has been received by editors: 03.01.2019.

The paper has been received by editors after peer-review: 18.01.2019.

The paper has been accepted for publication: 31.01.2019. 


\title{
Закономерности блочного подхода для анализа структуры химических элементов и проблемы материаловедения
}

\author{
Авторы: \\ Гусев Борис Владимирович, \\ зав. кафедрой «Строительные материалы и технологии» \\ Российского университета транспорта, Москва, Россия, e-mail: info-rae@mail.ru \\ Сперанский Анатолий Алексеевич, \\ вице-президент РИА, Москва, Россия, e-mail: vibro-vector@yandex.ru
}

Резюме: Авторами разработано представление об объемной матрице химических элементов, которое позволило устранить основные недостатки табличных форм структуры химических элементов, разработанных Д.И. Менделеевым и международным сообществом химиков ИЮПАК. Объемная структура представлена в виде расширяющей конусной матрицы. При этом все известные химические элементы до номера 118 объединены в четыре блока. Представление о блочности позволило обосновать электронно-уровневую формулу, в том числе для предполагаемых новых химических элементов 5-го блока $\mathrm{E}$ с номерами 119 по 218. Это позволяет разработать цифровую модель для расчета взаимодействий химических элементов и получения новых видов соединений и материалов.

Ключевые слова: химический элемент, табличные формы периодической структуры, объемная матрица, механизмы периодичности, орбитальные модели электронных оболочек атомов.

Для цитирования: Гусев Б.В., Сперанский А.А. Закономерности блочного подхода для анализа структуры химических элементов и проблемы материаловедения // Нанотехнологии в строительстве. - 2019. - Том 11, № 1. - C. 76-88. - DOI: 10.15828/2075-8545-2019-11-1-76-88.

Machine-readable information on CC-licenses (HTML-code) in metadata of the paper

$<$ a rel="license" href="http://creativecommons.org/licenses/by/4.0/" $><$ img alt="Creative Commons License" style="border-width:0" src="https://i.creativecommons.org/l/by/4.0/88x31.png" / ></a $><$ br $/><$ span xmlns:dct="http://purl.org/dc/terms/" href="http://purl.org/dc/dcmitype/Text" property="dct:title" rel="dct:type" $>$ Patterns of the block approach for structure analysis of chemical elements and issues in materials science $</$ span $>$ by $<$ a xmlns:cc="http://creativecommons.org/ns\#" href="Nanotehnologii v stroitel'stve = Nanotechnologies in Construction. 2019, Vol. 11, no. 1, pp. 76-88. DOI: 10.15828/2075-8545-2019-11-1-76-88" property="cc:attributionName" rel="cc:attributionURL" $>$ Gusev B.V., Speransky A.A. $</ a>$ is licensed under a $<$ a rel="license" href="http://creativecommons.org/licenses/ by/4.0/" $>$ Creative Commons Attribution 4.0 International License $</ \mathrm{a}>.<\mathrm{br} />$ Based on a work at $<$ a xmlns:dct="http://purl.org/dc/terms/" href="http://nanobuild.ru/ en_EN/nanobuild-1-2019/" rel="dct:source" $>$ http://nanobuild.ru/en_EN/nanobuild-1-2019/</a $>.<b r />$ Permissions beyond the scope of this license may be available at $<$ a xmlns:cc="http://creativecommons.org/ns\#" href="info-rae@mail.ru" rel="cc:morePermissions" $>$ info-rae@mail.ru $</ a>$.

Статья поступила в редакцию: 03.01.2019.

Статья поступила в редакцию после рецензирования: 18.01.2019.

Статья принята к публикации: 31.01.2019. 


\section{INTRODUCTION}

In January 2019, the World community celebrated the $150^{\text {th }}$ anniversary of one of the greatest discoveries in the field of fundamental scientific knowledge - the publication of the periodic table of chemical elements of D.I. Mendeleev, and it seems relevant to express some considerations related to modern tasks of scientific and technological development.

In the structural materials science, the main task of which is to obtain a substance with high performance characteristics, it is necessary to turn to the scientific basis of natural sciences - the periodic law of D.I. Mendeleev. The author of the law himself repeatedly returned to the issues of its «incompleteness and practical ambiguities, including the reasons for the law of multiple relationships, differences in elements and changes in their atomicity, and at the same time it is necessary to understand what is mass and aggravation» [1]. And, if the first part of the problem is practically solved by the science of matter, then the understanding of the second part is still relevant.

\section{MAIN PART}

An important list of questions in terms of improving knowledge about periodicity was voiced by Nobel Prize Laureate Academician N.N. Semyonov in a stage lecture in 1951 to the students of the physics faculty: «The goal is clear - after eliminating shortcomings, new properties and interrelationships of elements will be revealed, which will allow most scientific research at a new, higher level, and solving engineering problems not only to you - engineersphysicists, but to all scientists, engineers and practitioners. So far no one in the world has managed to eliminate these obvious shortcomings! And this is necessary for advancing science, technology, and engineering». The main shortcomings of the periodic table were the following:

1. Rows (the so-called half-periods) in the designated periods have different lengths. The number of free cells is 37 .

2. There are only two elements in the first row; moreover, hydrogen does not occupy a permanent place, and these two elements make up a whole period.

3. Lanthanides and Actinides were outside the table.

4. The group of inert gases discovered subsequently by scientists was added.

5. The position introduced later in the long-period table as a whole does not help the issue, the table continues to remain asymmetric. There are 7 periods, and 10 rows, that is, a half-period and a series are different mismatched concepts [2].

Many scientists and practitioners from different countries of the world, from well-known chemists to persistent practitioners, have made efforts to solve the proclaimed problem. From the numerous unsuccessful attempts to improve the periodic table, there follows the genius of D.I. Mendeleev and the extreme complexity of the task set by Academician Semyonov, the urgency of which is not questioned. At the same time, the universal striving for scientific and technical leadership in mastering the sixth technological order observed in the world economy indicates that informational modernization of the system of chemical elements is a subject of great importance [3].

This article presents a three-dimensional matrix of chemical elements as an identification-analytical tool that allows you to study a wide variety of physicochemical properties of already known and not yet open elements through the construction of dynamic energy models of the electronic shells of the elements themselves and their compounds. The universality of the volume matrix lies in the fact that in addition to the required sequence number and strict coordinate binding of chemical elements (or their cluster formations) to groups, there are wide possibilities for structural analysis of the physicochemical properties of elements, laws and regularities of their interactions using periodic orbital structures [3].

When studying the problems of periodicity, rather obvious assumptions were made. The world is multidimensional and is typically considered in spatial dimensions, and the table is two-dimensional. Furthermore, a more convincing idea was formulated of considering chemical elements from the position of their origin as materials of the Universe (atoms are stellar matter). During the formation and development of the Universe, at the first stage only hydrogen and helium existed and they should be at the head of the table or, more figuratively, of the three-dimensional matrix. Then light elements appeared and only stars with their high temperatures and pressures could synthesize heavy nuclei. The next assumption was that the creation of elements also took place in a spiral, as the Universe develops [4].

The three-dimensional matrix presents the indexes of chemical elements in the form of a continuous series of natural numbers from 1 to 118 and further, evenly distributed in a spiral from top to bottom (Fig. 1). The index number of the element coincides with the value of the nuclear charge and the same total number of energetically balanced electrons in the orbitals. The approach is universal with respect to both the short-term table of D.I. Mendeleev and the long-period IUPAC table.

The 3D-spiral matrix of chemical elements has 4 periodicity blocks [3]:

- in the first block A, the first elements of the emerging Universe are hydrogen and helium;

- the second block B consists of two identical periods of eight elements from lithium to argon;

- in block C, two additional cluster formations appeared: iron, cobalt, nickel and ruthenium, rhodium, palladium; 




Fig. 1. Three-dimensional periodic matrix of chemical elements 
- in block D, the lanthanide and actinide families were additionally included, as well as two clusters: osmium, iridium, platinum, and hassium, maytnerium, and darmstadtium.

The form of the three-dimensional periodic matrix allowed structuring the anomalous families of the third (lanthanides and actinoids) and eighth (metalloids) groups of the third $\mathrm{C}$ and fourth $\mathrm{D}$ levels of block periodicity. In addition, the level formulas of electronic shells facilitate the formation of a model for studying the system regularities of periodicity, including saturation mechanisms and the interlevel valence transition through the distribution of electrons over different energy levels (orbitals, states) of the electron shells. The consistency of periodicity is relevant to the study of subtle mechanisms of interlevel transitions and stable failures of the synthesis of electronic orbitals.

In connection with the anniversary of the publication of the fundamental discovery of D.I. Mendeleev, it is appropriate to consider four levels of knowledge of the periodic law.
The first level corresponds to a generalization of the facts of changes in chemical properties depending on the structure of the nucleus and the increase in atomic masses.

The second level of knowledge corresponds to the introduction of the concept of periodicity of electron shell structure of atoms, which energetically balances the nuclear charge and demonstrates the repetition of similar types of electronic configurations of atoms as the atomic number (index number) increases.

The third level of knowledge is associated with the development of a periodic structure of electron-level orbitals of shells in the system of atomic energy levels, realized as a model of atomic-energy (valent) interaction.

The fourth level will make it possible to form methods and conditions for the formation of models of substances, taking into account the energy interactions of the chemical elements involved in this process.

The concept of block structure properties, which allows predicting chemical elements beyond the $118^{\text {th }}$ element based on electron-level formulas, should also be at-

Table 1

Energy levels of electron shells of chemical elements

\begin{tabular}{|l|l|l|l}
\hline s - elements & $p$ - elements & $d$ - elements & $f$ - elements
\end{tabular}

\begin{tabular}{|c|c|c|c|}
\hline \multicolumn{3}{|c|}{$\begin{array}{l}\text { No, denotation, } \\
\text { Name of the elements }\end{array}$} & $\begin{array}{l}\text { Short electron STRUCTURE of energy shell completion in the atoms of } \\
\text { chemical elements }\end{array}$ \\
\hline \multicolumn{3}{|c|}{ The short block $A$} & $f^{\prime \prime}$ energy level $\underline{K^{\circ}}$ \\
\hline 1 & $\mathrm{H}$ & Hydrogen & $K^{1}$ \\
\hline 2 & $\mathrm{He}$ & Helium & $\underline{K}^{2} 1^{\text {st }}$ complete energy level $\underline{K}^{2} \equiv 1 \mathrm{~s}^{2}$ \\
\hline
\end{tabular}

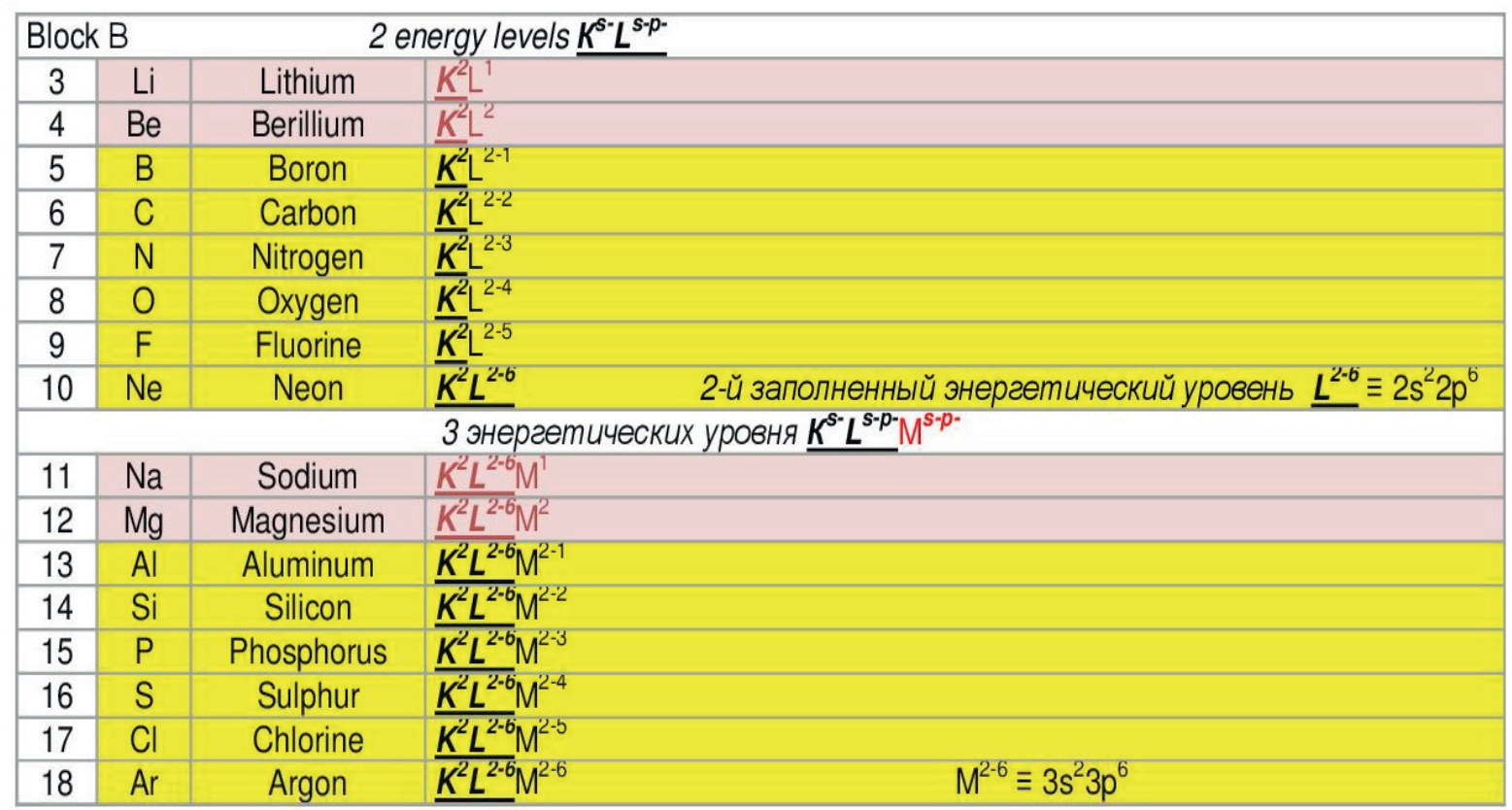


Continuation of Table 1

\begin{tabular}{|c|c|c|c|}
\hline \multicolumn{2}{|c|}{ Block C } & \multicolumn{2}{|c|}{4 energy levels $K^{S-L^{s-p-}} M^{s-p-d-} N^{s-p-}$} \\
\hline 19 & K & Potassium & $K^{2} L^{2-6} M^{2-6} N^{1}$ \\
\hline 20 & $\mathrm{Ca}$ & Calcium & $K^{2} L^{2-6} M^{2-6} N^{2}$ \\
\hline 21 & Sc & Scandium & $K^{2} L^{2-6} M^{2-6-1} N^{2}$ \\
\hline & & & $\downarrow$ \\
\hline 30 & $\mathrm{Zn}$ & Zinc & 3-й заполненный уровень $\underline{M^{2-6-10}} \equiv 3 s^{2} 3 p^{6} 3 d^{10}$ \\
\hline 31 & $\mathrm{Ga}$ & Gallium & $K^{2} L^{2-6} M^{2-6-10} N^{2-1}$ \\
\hline 32 & $\mathrm{Ge}$ & Germanium & $K^{2} L^{2-6} M^{2-6-10} N^{2-2}$ \\
\hline 33 & As & Arsenic & $K^{2} L^{2-6} M^{2-6-10} N^{2-3}$ \\
\hline 34 & $\mathrm{Se}$ & Selenium & $K^{2} L^{2-6} M^{2-6-10} N^{2-4}$ \\
\hline 35 & $\mathrm{Br}$ & Bromine & $K^{2} L^{2-6} M^{2-6-10} N^{2-5}$ \\
\hline 36 & $\mathrm{Kr}$ & Krypton & $K^{2} L^{2-6} M^{2-6-10} N^{2-6}$ \\
\hline & & & 5 energy levels $K^{S-}-L^{s-p-} M^{s-p-d-} N^{s-p-d-} O^{s-p-}$ \\
\hline 37 & $\mathrm{Rb}$ & Rubidium & $K^{2} L^{2-6} M^{2-6-10} N^{2-6} O^{1}$ \\
\hline 38 & $\mathrm{Sr}$ & Strontium & $K^{2} L^{2-6} M^{2-6-10} N^{2-6} O^{2}$ \\
\hline 39 & Y & Yttrium & $K^{2} L^{2-6} M^{2-6-10} N^{2-6-1} O^{2}$ \\
\hline & & & $\downarrow$ \\
\hline 48 & $\mathrm{Cd}$ & Cadmium & $K^{2} L^{2-6} M^{2-6-10} N^{2-6-10} O^{2}$ \\
\hline 49 & In & Indium & $K^{2} L^{2-6} M^{2-6-10} N^{2-6-10} O^{2-1}$ \\
\hline 50 & Sn & Tin & $K^{2} L^{2-6} M^{2-6-10} \mathrm{~N}^{2-6-10} \mathrm{O}^{2-2}$ \\
\hline 51 & $\mathrm{Sb}$ & Antimony & $K^{2} L^{2-6} M^{2-6-10} N^{2-6-10} O^{2-3}$ \\
\hline 52 & $\mathrm{Te}$ & Tellirium & $K^{2} L^{2-6} M^{2-6-10} N^{2-6-10} O^{2-4}$ \\
\hline 53 & 1 & lodine & $K^{2} L^{2-6} M^{2-6-10} N^{2-6-10} O^{2-5}$ \\
\hline 54 & $\mathrm{Xe}$ & Xenon & $K^{2} L^{2-6} M^{2-6-10} N^{2-6-10} O^{2-6}$ \\
\hline
\end{tabular}

\begin{tabular}{|c|c|c|c|}
\hline \multicolumn{3}{|c|}{ block D } & \multirow{2}{*}{ 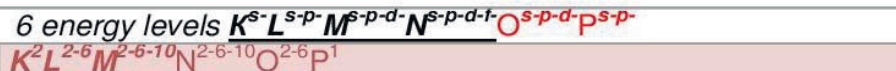 } \\
\hline 55 & Cs & Cesium & \\
\hline 56 & $\mathrm{Ba}$ & Barium & $K^{2} L^{2-6} M^{2-6-10} N^{2-6-10} O^{2-6} P^{2}$ \\
\hline 57 & $\mathrm{La}$ & Lanthanum & $K^{2} L^{2-6} M^{2-6-10} N^{2-6-10} O^{2-6-1} P^{2}$ \\
\hline 70 & $\mathrm{Yb}$ & Ytterbium & $K^{2} L^{2-6} M^{2-6-10} N^{2-6-10-14} O^{2-6} P^{2}$ \\
\hline 71 & Lu & Lutetium & $K^{2} L^{2-6} M^{2-6-10} N^{2-6-10-14} O^{2-6-1} P^{2}$ \\
\hline & & & $K^{2} 2^{2-6} M^{2-6-10} N^{2-6-10-14} \stackrel{1}{\frac{1}{2}} \mathrm{C}^{2-6-10} \mathrm{P}^{2}$ \\
\hline $\begin{array}{l}80 \\
81\end{array}$ & $\frac{\mathrm{Hg}}{\mathrm{Tl}}$ & $\begin{array}{l}\text { Mercury } \\
\text { Thallium }\end{array}$ &  \\
\hline 82 & $\mathrm{~Pb}$ & Lead & $K^{2} L^{2-6} M^{2-6-10} N^{2-6-10-14} O^{2-6-10} P^{2-2}$ \\
\hline 83 & $\mathrm{Bi}$ & Bismuth & $K^{2} L^{2-6} M^{2-6-10} N^{2-6-10-14} O^{2-6-10} P^{2-3}$ \\
\hline 84 & Po & Polonium & $K^{2} L^{2-6} M^{2-6-10} N^{2-6-10-14} O^{2-6-10} P^{2-4}$ \\
\hline 85 & At & Astatine & $K^{2} L^{2-6} M^{2-6-10} N^{2-6-10-14} O^{2-6-10} P^{2-5}$ \\
\hline 86 & $\mathrm{Rn}$ & Radon & $K^{2} L^{2-6} M^{2-6-10} N^{2-6-10-14} O^{2-6-10} P^{2-6}$ \\
\hline & & & 7 energy levels $K^{s-L^{s-p}-} M^{s-p-d-} N^{s-p-d}$ \\
\hline 87 & $\mathrm{Fr}$ & Francium & $K^{2} L^{2-6} M^{2-6-10} N^{2-6-10-14} O^{2-6-10} P^{2-6} Q^{1}$ \\
\hline 88 & $\mathrm{Ra}$ & Radium & $K^{2} L^{2-6} M^{2-6-10} N^{2-6-10-74} O^{2-6-10} P^{2-6} Q^{2}$ \\
\hline 89 & Ac & Actinium & $K^{2} L^{2-6} M^{2-6-10} N^{2-6-10-14} O^{2-6-10} P^{2-6-1} Q^{2}$ \\
\hline & & & $\pm \frac{1}{1}$ \\
\hline 103 & $\mathrm{Lr}$ & Lawrencium & $\underline{K^{2} L^{2-6} M^{2-6-10} N^{2-6-10-14} O^{2-6-10-14} P^{2-6-1} Q^{2}}$ \\
\hline & & & \\
\hline 112 & $\mathrm{Cn}$ & Copernicium & 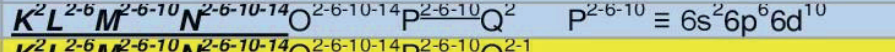 \\
\hline 113 & $\mathrm{Nh}$ & Nihonium & $K^{2} L^{2-6} M^{2-6-10} N^{2-6-10-14} \mathrm{O}^{2-6-10-14} \mathrm{P}^{2-6-10} \mathrm{Q}^{2-1}$ \\
\hline 114 & $\mathrm{FI}$ & Flerovium & $K^{2} L^{2-6} M^{2-6-10} N^{2-6-10-14} O^{2-6-10-14} \mathrm{P}^{2-6-10} \mathrm{Q}^{2-2}$ \\
\hline 115 & Mc & Moscovium & $K^{2} L^{2-6} M^{2-6-10} N^{2-6-10-74} O^{2-6-10-14} P^{2-6-10} Q^{2-3}$ \\
\hline 116 & Lv & Livermorium & $K^{2} L^{2-6} M^{2-6-10} N^{2-6-10-14} O^{2-6-10-14} P^{2-6-10} Q^{2-4}$ \\
\hline 117 & Ts & Tennessine & $K^{2} L^{2-6} M^{2-6-10} N^{2-6-10-14} O^{2-4}$ \\
\hline 118 & Og & Oganesson & $-14 P^{2-6-10} Q^{2-6} \quad Q^{2-6} \equiv 7 s^{2} 7 p^{6}$ \\
\hline
\end{tabular}


Continuation of Table 1

\begin{tabular}{|c|c|c|c|}
\hline \multicolumn{3}{|c|}{ block E } & \multirow{2}{*}{$\begin{array}{l}8 \text { энергетических уровней } \frac{K^{s-}-L^{s-p-}-M^{s-p-d-} N^{s-p-d-f-} O^{s-p-d-f-g-} P^{s-p-d-f-} Q^{s-p-d-} X^{s-p-}}{K^{2} L^{2-6} M^{2-6-10} N^{2-6-10-14} O^{2-6-10-14} P^{2-6-10} Q^{2-6} X^{1}}\end{array}$} \\
\hline 119 & $s-1$ & s-orbital & \\
\hline 120 & $s-2$ & s-orbital & $K^{2} L^{2-6} M^{2-6-70} N^{2-6-10-74} O^{2-6-10-14} P^{2-6-10} Q^{2-6} X^{2}$ \\
\hline 121 & $g-1$ & $g$ - orbital & $K^{2} L^{2-6} M^{2-6-10} N^{2-6-10-14} O^{2-6-10-14-1} P^{2-6-10} Q^{2-6} X^{2}$ \\
\hline & & & 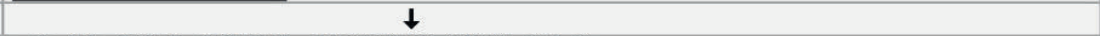 \\
\hline 138 & $g-18$ & $\boldsymbol{g}$ - orbital & $K^{2} L^{2-6} M^{2-6-10} N^{2-6-10-14} O^{2-6-10-14-18} \mathrm{P}^{2-6-10} \mathrm{Q}^{2-6} \mathrm{X}^{2}$ \\
\hline 139 & $f-1$ & $f$ - orbital & $K^{2} L^{2-6} M^{2-6-10} N^{2-6-10-14} O^{2-6-10-74-18} \mathrm{P}^{2-6-10-1} \mathrm{Q}^{2-6} \mathrm{X}^{2}$ \\
\hline & & & $\downarrow$ \\
\hline 152 & $f-14$ & $\boldsymbol{f}$ - orbital & $K^{2} L^{2-6} M^{2-6-10} N^{2-6-10-14} O^{2-6-10-14-18} \mathrm{P}^{2-6-10-14} Q^{2-6} X^{2}$ \\
\hline 153 & $d-1$ & d-orbital & $K^{2} L^{2-6} M^{2-6-10} N^{2-6-10-14} O^{2-6-10-14-18} \mathrm{P}^{2-6-10-14} \mathrm{Q}^{2-6-1} \mathrm{X}^{2}$ \\
\hline & & & $\downarrow$ \\
\hline 162 & $d-10$ & $\boldsymbol{d}$ - orbital & $K^{2} L^{2-6} M^{2-6-10} N^{2-6-10-14} O^{2-6-10-14-18} \mathrm{P}^{2-6-10-14} \mathrm{Q}^{2-6-10} \mathrm{X}^{2}$ \\
\hline 163 & $p-1$ & $\boldsymbol{p}$ - orbital & $K^{2} L^{2-6} M^{2-6-10} N^{2-6-10-14} O^{2-6-10-14-18} \mathrm{P}^{2-6-10-14} \mathrm{Q}^{2-6-10} \mathrm{X}^{2-1}$ \\
\hline 164 & $p-2$ & $\boldsymbol{p}$ - orbital & $K^{2} L^{2-6} M^{2-6-10} N^{2-6-10-14} O^{2-6-10-14-18} \mathrm{P}^{2-6}$ \\
\hline 165 & $p-3$ & $\boldsymbol{p}$ - orbital & $K^{2} L^{2-6} M^{2-6-10} N^{2-6-10-14} O^{2-6-10-14-18} \mathrm{P}^{2-}$ \\
\hline 166 & $p-4$ & p- orbital & $K^{2} L^{2-6} M^{2-6-10} N^{2-6-10-14} O^{2-6-10-14-18} \mathrm{P}^{2-6-10-14} \mathrm{Q}^{2-6-10} \mathrm{X}^{2-4}$ \\
\hline 167 & $p-5$ & p-orbital & $K^{2} L^{2-6} M^{2-6-10} N^{2-6-70-14} O^{2-6-10-14-18} \mathrm{P}^{2-6-10-14} \mathrm{Q}^{2-6-10} \mathrm{X}^{2-5}$ \\
\hline 168 & $p-6$ & p-orbital & $K^{2} L^{2-6} M^{2-6-10} N^{2-6-10-14} O^{2-6-10-14-18} \mathrm{P}^{2-6-10-14} \mathrm{Q}^{2-6-10} \mathrm{X}^{2-6}$ \\
\hline \multicolumn{4}{|c|}{9 энергетических уровней $\underline{K}^{s-}-L^{s-p-}-M^{s-p-d-} N^{s-p-d-1-} O^{s-p-d-1-g-} P^{s-p-d-1-g-} Q^{s-p-d-1-} X^{s-p-d-} Y^{s-p-}$} \\
\hline 169 & $s-1$ & s-orbital & $K^{2} L^{2-6} M^{2-6-10} N^{2-6-10-14} O^{2-6-10-14-18} \mathrm{P}^{2-6-10-14} \mathrm{Q}^{2-6-10} \mathrm{X}^{2-6} \mathrm{Y}^{1}$ \\
\hline 170 & $s-2$ & s-orbital & $K^{2} L^{2-6} M^{2-6-10} N^{2-6-10-14} O^{2-6-10-14-18} \mathrm{P}^{2-6-10-14} \mathrm{Q}^{2-6-10} \mathrm{X}^{2-6} \mathrm{Y}^{2}$ \\
\hline \multirow[t]{2}{*}{171} & $g-1$ & $\boldsymbol{g}$ - orbital & $K^{2} L^{2-6} M^{2-6-10} N^{2-6-10-14} O^{2-6-10-14-18} \mathrm{P}^{2-6}$ \\
\hline & & & $\downarrow$ \\
\hline 188 & $g-18$ & $\boldsymbol{g}$ - orbital & $K^{2} L^{2-6} M^{2-6-10} N^{2-6-10-14} O^{2-6-10-14-18} \mathrm{P}^{2-6-10-14-18} \mathrm{Q}^{2-6-10} \mathrm{X}^{2-6} \mathrm{Y}^{2}$ \\
\hline \multirow[t]{2}{*}{189} & $f-1$ & $\boldsymbol{f}$ - orbital & $K^{2} L^{2-6} M^{2-6-10} N^{2-6-10-14} O^{2-6-10-14-18} \mathrm{P}^{2-6-1}$ \\
\hline & & & $\downarrow$ \\
\hline 202 & $f-14$ & $\boldsymbol{f}$ - orbital & $K^{2} L^{2-6} M^{2-6-10} N^{2-6-10-14} O^{2-6-10-14-18} \mathrm{P}^{2-6-}$ \\
\hline \multirow[t]{2}{*}{203} & $d-1$ & $\boldsymbol{d}$ - orbital & $K^{2} L^{2-6} M^{2-6-10} N^{2-6-70-14} O^{2-6-10-14-18} \mathrm{P}^{2-6}$ \\
\hline & & & \\
\hline 212 & $d-10$ & $\boldsymbol{d}$ - orbital & $K^{2} L^{2-6} M^{2-6-10} N^{2-6-10-14} O^{2-6-10-14-18} \mathrm{P}^{2-6-10-14-18} \mathrm{Q}^{2-6-10-14} \mathrm{X}^{2-6-10} \mathrm{Y}^{2}$ \\
\hline 213 & $p-1$ & p-orbital & $K^{2} L^{2-6} M^{2-6-10} N^{2-6-10-14} O^{2-6-10-14-18} \mathrm{P}^{2-6-10-14-18} \mathrm{Q}^{2-6-10-14} \mathrm{X}^{2-6-10} \mathrm{Y}^{2-1}$ \\
\hline 214 & $p-2$ & $p$ - orbital & $K^{2} L^{2-6} M^{2-6-10} N^{2-6-10-14} O^{2-6-10-14-18} \mathrm{P}^{2-6-}$ \\
\hline 215 & $p-3$ & p- orbital & $K^{2} L^{2-6} M^{2-6-10} N^{2-6-10-14} O^{2-6-10-14-18} \mathrm{P}^{2-6-10-14-18} \mathrm{Q}^{2-6-10-14} \mathrm{X}^{2-6-10} \mathrm{Y}^{2-3}$ \\
\hline 216 & $p-4$ & p-orbital & $K^{2} L^{2-6} M^{2-6-10} N^{2-6-10-14} O^{2-6-70-14-18} \mathrm{P}^{2-6-10-14-18} \mathrm{Q}^{2-6-10-14} \mathrm{X}^{2-6-10} \mathrm{Y}^{2-4}$ \\
\hline 217 & $p-5$ & p-orbital & $K^{2} L^{2-6} M^{2-6-10} N^{2-6-10-14} O^{2-6-10-14-18} \mathrm{P}^{2-6-1}$ \\
\hline 218 & $p-6$ & $\boldsymbol{p}$ - orbital & $K^{2} L^{2-6} M^{2-6-10} N^{2-6-10-14} O^{2-6-10-14-18} P^{2-6-10-14-18} Q^{2-6-10-14} X^{2-6-10} Y^{2-6}$ \\
\hline
\end{tabular}

tributed to this. Tab. 1 shows the electron-orbital formulas of chemical elements for all blocks, including the fifth $\mathrm{E}$ block, which covers elements from 119 to 218 .

From the standpoint of physics, it is advisable to consider the result of energy interactions that determine all the properties of a substance. There may be two approaches that define two energy models of the structural representation of matter. One model using electronorbital formulas (formal) (Table 1), and another model (physical) from the standpoint of structuring based on quantum phenomena and physical constants.

The physical method is different from the formal one in that it is based on the causal correspondence of the energy interactions of the physical fields (cause) of the space-time position of the electrons of the shells of ho- mogeneous and heterogeneous chemical elements (consequence). This situation is similar to the concepts of classical mechanics and can be determined by a number of constants [4].

\section{CONCLUSION}

Further research on the formation of substance structures and the assessment of its influence on all types of interactions are the strong and weak electromagnetic and gravitational fields, which will be characterized by fundamental physical constants. The authors will continue research on the consideration of those constants that affect the processes of structure formation and the creation of new types of structural materials. 


\section{ВВЕДЕНИЕ}

$\mathrm{B}$ январе 2019 года Мировое сообщество отметило 150-летие величайшего открытия в области фундаментальных научных знаний - опубликования периодической таблицы химических элементов Д.И. Менделеева - и представляется актуальным высказать некоторые соображения, связанные с современными задачами научно-технического развития.

В конструкционном материаловедении главной задачей является получение вещества с высокими эксплуатационными характеристиками, и неизбежно приходится обращаться к научной основе естествознания, периодическому закону Д.И. Менделеева. Сам автор выдающегося закона неоднократно возвращался к вопросам его «незавершенности и практических неясностей, в том числе к причинам закона кратных отношений, различия элементов и изменения их атомности, и в то же время нужно понять, что такое масса и тяготение» [1]. И если первая часть задачи наукой о веществе практически решена, то понимание второй её части актуально до сих пор.

\section{ОСНОВНАЯ ЧАСТЬ}

Важный перечень вопросов в плане совершенствования знаний о периодичности озвучил лауреат Нобелевской премии академик Н.Н.Семёнов в постановочной лекции 1951 года перед студентами Физтеха: «Цель ясна - после устранения недостатков обнаружатся новые свойства и взаимосвязи элементов, что позволит проводить большинство научных исследований на новом, более высоком уровне и решать стоящие инженерные задачи не только вам - инженерам-физикам, а всем ученым, инженерам и практикам. Устранить эти очевидные недостатки пока никому в мире не удалось! А это необходимо для продвижения науки, технологий, техники и инженерии вперед». Озвучены главные недостатки периодической таблицы:

1. Ряды (так называемые полупериоды) в обозначенных периодах имеют разную длину. При этом число свободных мест - клеток 37.

2. В первом ряду элементов всего два; к тому же водород не занимает постоянного места, а эти два элемента составляют целый период.

3. Лантаноиды и Актиноиды оказались за пределами таблицы.

4. Добавилась открытая впоследствии учеными группа инертных газов.

5. Введенная позже длиннопериодная таблица положения в целом не спасает, таблица продолжает оставаться асимметричной. Периодов 7, а рядов 10, то есть полупериод и ряд - разные несовпадающие понятия [2].
К разрешению провозглашенной проблемы приложили усилия многие ученые и практики из разных стран мира, от известных химиков до настойчивых практиков. Из многочисленных безуспешных попыток усовершенствовать периодическую таблицу следует гениальность Д.И. Менделеева и чрезвычайная сложность поставленной академиком Н.Н. Семёновым задачи, актуальность которой никто не ставит под сомнение. Вместе с тем, наблюдаемое в мировой экономике всеобщее стремление к научно-техническому лидерству в освоении VI-го технологического уклада свидетельствует о том, что информационная модернизация системы химических элементов является предметом большой значимость [3].

В настоящей статье представлена объемная матрица химических элементов в качестве идентификационно - аналитического инструмента, который позволяет изучать большое многообразие физикохимических свойств уже известных и еще не открытых элементов через построение динамических энергетических моделей электронных оболочек собственно элементов и их соединений. Универсальность объемной матрицы состоит в том, что помимо обязательного порядкового номера и строгой координатной привязки химических элементов (либо их кластерных образований) к группам имеются широкие возможности структурного анализа физико-химических свойств элементов, законов и закономерностей их взаимодействий с использованием орбитальных структур периодичности [3].

При изучении проблем периодичности в качестве идеи были сформулированы достаточно очевидные положения. Мир многомерен и, как правило, рассматривается в пространственных измерениях, а таблица - двухмерная. Далее была сформулирована более убедительная идея рассмотрения химических элементов с позиции их происхождения в качестве материалов Вселенной (атомы - это звездная материя). При образовании и развитии Вселенной на первом этапе существовали только водород и гелий, и они должны быть во главе таблицы или, что более образно, объемной матрицы. Затем возникли легкие элементы, и только звезды с их высокими температурами и давлениями могли синтезировать тяжелые ядра. В качестве следующего предположения было принято, что создание элементов так же происходило по спирали, как развивается Вселенная [4].

На объемно-каркасной матрице представлены номера химических элементов в виде непрерывного ряда натуральных чисел от 1 до 118 и далее, равномерно распределенных по спирали сверху вниз (рис. 1). Порядковый номер элемента совпадает с величиной заряда ядра и таким же суммарным количеством энергетически уравновешивающих электронов на орбиталях оболочек. Подход универсален как по 


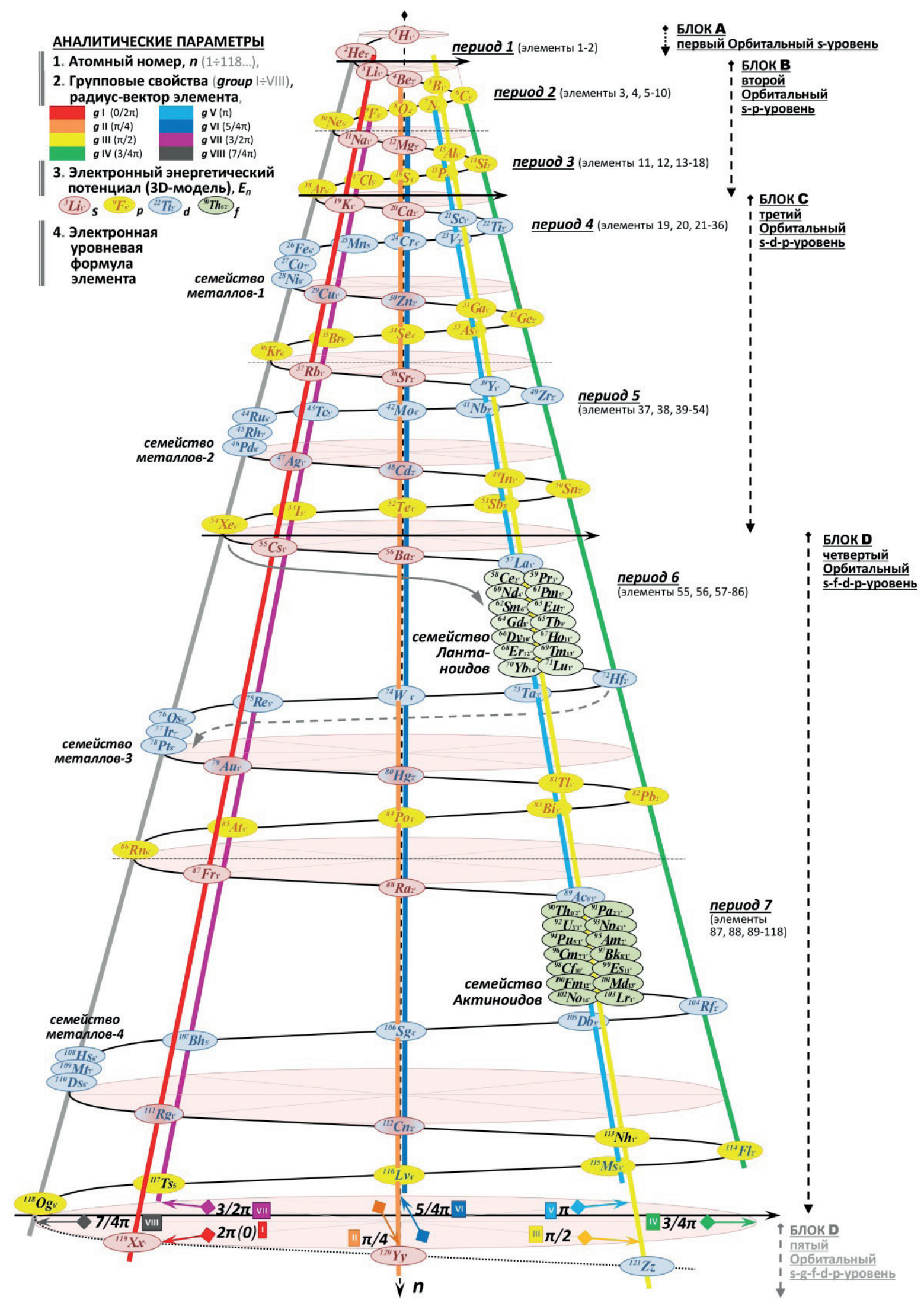

Рис.1. Объемная периодическая матрица химических элементов 
отношению к короткопериодной таблице Д.И. Менделеева, так и длиннопериодной таблице IUPAK.

3D-спирально пространственная расходящаяся система каркаса матрицы химических элементов имеет 4 блока периодичности [3]:

- в первом блоке А представлены первые элементы народившейся Вселенной, водород и гелий;

- второй блок В образуют два одинаковых периода из восьми элементов от лития до аргона;

- в блоке С появились два дополнительных кластерных образования: железо, кобальт, никель и рутений, родий, палладий;

- в блоке D дополнительно включились семейства лантаноидов и актиноидов, а также два кластера: осмий, иридий, платина и хассий майтнерий, дармштадтий.

Пространственная форма объемной периодической матрицы позволила структурировать аномальные семейства III-й (лантаноиды и актиноиды) и VIII-й (металлоиды) групп третьего С и четвертого D уровней блочной периодичности, а уровневые формулы электронных оболочек облегчают формирование модели изучения системных закономерностей периодичности, включая механизмы насыщения и межуровневого перехода валентности через распределение электронов по разным энергетическим уровням (орбитали, состояния) электронных оболочек. Системность периодичности актуальна при изучении тонких механизмов межуровневых переходов и устойчивых сбоев процесса синтеза электронных орбиталей.

В связи с международным юбилеем опубликования фундаментального открытия Д.И. Менделеева уместно рассматривать четыре уровня познания периодического закона.

Первый уровень соответствует обобщению фактов изменения химических свойств в зависимости от структуры ядра и возрастания атомных масс.

Второму уровню познания отвечает введение понятия о периодичности структуры электронной оболочки ядра атома, энергетически уравновешивающей заряд ядра и демонстрирующей повторение сходных типов электронных конфигураций атомов по мере увеличения атомного номера (порядкового номера элемента).

Третий уровень познания связан с разработкой периодической структуры электронно-уровневых орбиталей оболочек в системе энергетических уровней атома, реализующих в виде модели атомно-энергетического (валентного) взаимодействия.

Четвертый уровень позволит сформировать методы и условия формирования моделей веществ с учетом энергетических взаимодействий химических элементов, участвующих в этом процессе.

Таблица 1

Энергетические уровни электронных оболочек атомов химических элементов

$$
\begin{array}{|l|l|l|l|}
\hline \text { s-элементы } & \text { p-элементы } & \text { d-элементы } & \text { f-элементы } \\
\hline
\end{array}
$$

\section{№, химический знак, название элемента \\ краткая электронная СТРУКТУРА заполнения энергетических уровней электронных оболочек атомов химических элементов}

\begin{tabular}{|c|c|c|c|}
\hline \multicolumn{3}{|c|}{ блок А короткий } & 1энергетический уровень $K^{\circ}$ \\
\hline 1 & $\mathrm{H}$ & Водород & 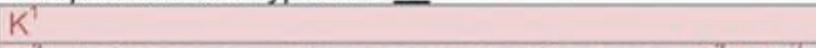 \\
\hline 2 & $\mathrm{He}$ & Гелий & $\underline{K}^{2}$ 1-й заполненный энергетический уровень $\underline{K}^{2} \equiv 1 \mathrm{~s}^{2}$ \\
\hline
\end{tabular}

\begin{tabular}{|c|c|c|c|}
\hline \multicolumn{3}{|c|}{ блок В } & \multirow{2}{*}{2 энергетических уровня $K^{5 \cdot L^{\text {s.p. }}}$} \\
\hline 3 & $\mathrm{Li}$ & Литий & \\
\hline 4 & $\mathrm{Be}$ & Бериллий & $K^{2} L^{2}$ \\
\hline 5 & $\mathrm{~B}$ & Бop & $\underline{K}^{2} L^{2-1}$ \\
\hline 6 & C & Углерод & $\underline{K}^{2} L^{2-2}$ \\
\hline 7 & $\mathrm{~N}$ & Азот & $\bar{K}^{2} L^{2-3}$ \\
\hline 8 & 0 & Кислород & $K^{2} L^{2-4}$ \\
\hline 9 & $\mathrm{~F}$ & Фтор & $\underline{K}^{2} L^{2-5}$ \\
\hline 10 & $\mathrm{Ne}$ & $\mathrm{HeOH}$ & 2-й заполненный энергетический уровень $\underline{L}^{2-6} \equiv 2 \mathrm{~s}^{2} 2 \mathrm{p}^{6}$ \\
\hline \multicolumn{4}{|r|}{ 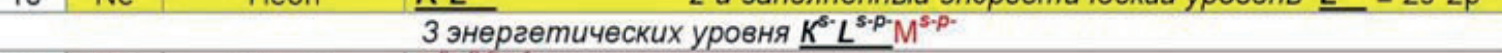 } \\
\hline 11 & $\mathrm{Na}$ & Натрий & $K^{2} L^{2-6} M^{1}$ \\
\hline 12 & $\mathrm{Mg}$ & Магний & $K^{2} L^{2-8} M^{2}$ \\
\hline 13 & $\mathrm{Al}$ & Алюминий & $K^{2} L^{2-6} M^{2-1}$ \\
\hline 14 & $\mathrm{Si}$ & Кремний & $K^{2} L^{2-6} M^{2-2}$ \\
\hline 15 & $\mathrm{P}$ & Фосфор & $K^{2} L^{2-6} M^{2-3}$ \\
\hline 16 & S & Cepa & $K^{2} L^{2-6} M^{2-4}$ \\
\hline 17 & $\mathrm{Cl}$ & Хлор & $K^{2} L^{2-6} M^{2-5}$ \\
\hline 18 & $\mathrm{Ar}$ & AрroH & $M^{2-6} \equiv 3 s^{2} 3 p^{6}$ \\
\hline
\end{tabular}


Продолжение таблицы 1

\begin{tabular}{|c|c|c|c|}
\hline \multicolumn{3}{|c|}{ блок C } & \multirow{2}{*}{$\begin{array}{l}4 \text { энергетических уровня } \underline{K}^{5 \cdot L^{s-p-} M^{s-p-d-} N^{s-p-}} \\
K^{2} L^{2-6} M^{2-5} N^{1}\end{array}$} \\
\hline 19 & K & Калий & \\
\hline 20 & $\mathrm{Ca}$ & Кальций & $K^{2} L^{2-6} M^{2-6} \mathrm{~N}^{2}$ \\
\hline \multirow[t]{2}{*}{21} & Sc & Скандий & \multirow{2}{*}{$\frac{K^{2} L^{2-6} M^{2-6-1} N^{2}}{\downarrow}$} \\
\hline & & & \\
\hline 30 & $\mathrm{Zn}$ & Циннк & 3-й заполненный уровень $\underline{M}^{2-6-10} \equiv 3 \mathrm{~s}^{2} 3 \mathrm{p}^{6} 3 \mathrm{~d}^{10}$ \\
\hline 31 & $\mathrm{Ga}$ & Галлий & $K^{2} L^{2-6} M^{2-6-10} N^{2-1}$ \\
\hline 32 & $\mathrm{Ge}$ & Германий & $K^{2} L^{2-6} M^{2-6-10} N^{2-2}$ \\
\hline 33 & As & Мышьяк & $K^{2} L^{2-6} M^{2-6-10} N^{2-3}$ \\
\hline 34 & $\mathrm{Se}$ & Селен & $K^{2} L^{2-6} M^{2-6-10} N^{2-4}$ \\
\hline 35 & $\mathrm{Br}$ & Бром & $K^{2} L^{2-6} M^{2-6-10} N^{2-5}$ \\
\hline 36 & $\mathrm{Kr}$ & Криптон & $K^{2} L^{2-8} M^{2-6-10} N^{2-8}$ \\
\hline \multicolumn{4}{|r|}{5 энераетических уровней $K^{s \cdot}-L^{s-p-} M^{s-p-\alpha} N^{s-p-\alpha \cdot} O^{s-p-}$} \\
\hline 37 & $\mathrm{Rb}$ & Рубидий & $K^{2} L^{2-6} M^{2-6-10} \mathrm{~N}^{2-6} \mathrm{O}^{1}$ \\
\hline 38 & $\mathrm{Sr}$ & Стронций & $K^{2} L^{2-6} M^{2-6.10} \mathrm{~N}^{2-6} \mathrm{O}^{2}$ \\
\hline 39 & $\mathrm{Y}$ & Иттрий & $K^{2} L^{2-6} M^{2-6-10} N^{2-6-1} O^{2}$ \\
\hline & & & 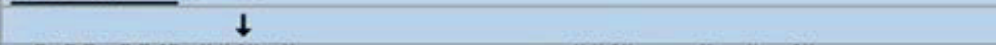 \\
\hline 48 & $\mathrm{Cd}$ & Кадмий & $K^{2} L^{2-6} M^{2-6-10} \mathrm{~N}^{2-6-10} \mathrm{O}^{2}$ \\
\hline 49 & In & Индий & $K^{2} L^{2-6} M^{2-6-10} N^{2-6-10} O^{2-1}$ \\
\hline 50 & Sn & Олово & $K^{2} L^{2-6} M^{2-6-10} N^{2-6-10} O^{2-2}$ \\
\hline 51 & $\mathrm{Sb}$ & Сурьма & $K^{2} L^{2-6} M^{2-6-10} N^{2-6-10} O^{2-3}$ \\
\hline 52 & $\mathrm{Te}$ & Теллур & $K^{2} L^{2-6} M^{2-6.70} N^{2-6-10} O^{2-4}$ \\
\hline 53 & 1 & Йод & $K^{2} L^{2-6} M^{2-6-10} N^{2-6-90} O^{2-5}$ \\
\hline 54 & $\mathrm{Xe}$ & Ксенон & $K^{2} L^{2-6} M^{2-6-10} N^{2-6-10} O^{2-6}$ \\
\hline
\end{tabular}

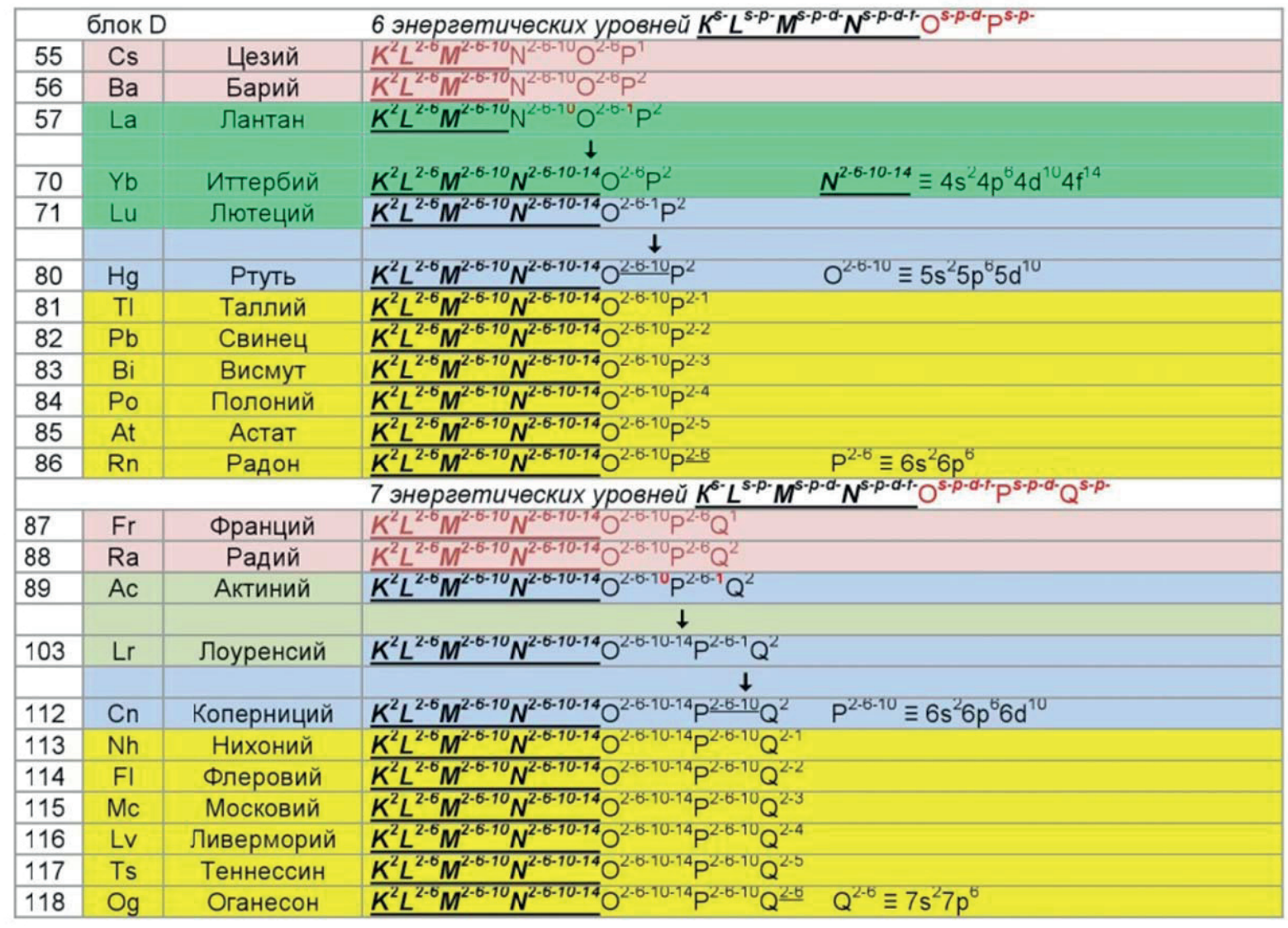


Nanotechnologies in Construction: A Scientific Internet-Journal Нанотехнологии в строительстве: научный Интернет-журнал
2019; $11(1):$ 76-88

STRUCTURING OF CHEMICALS ELEMENTS • СТРУКTУРИРОВАНИЕ ХИМИЧЕСКИХ ЭЛЕMEНTOВ

Окончание таблицы 1

\begin{tabular}{|c|c|c|c|}
\hline \multicolumn{3}{|c|}{ блок $\mathrm{E}$} & \multirow{2}{*}{ 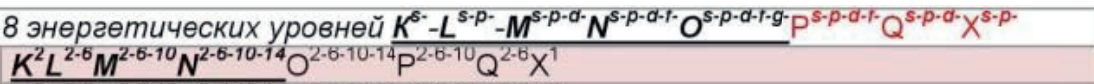 } \\
\hline 119 & $s-1$ & s-орбиталь & \\
\hline 120 & $s-2$ & s-орбиталь & $K^{2} L^{2-6} M^{2-6-10} N^{2-6-10-14} O^{2-6-10-14} P^{2-6-10} Q^{2-6} X^{2}$ \\
\hline 121 & $g-1$ & g- орбиталь & $K^{2} L^{2-6} M^{2-6-10} N^{2-6-10-14} O^{2-6-10-14-1} P^{2-6-10} Q^{2-6} X^{2}$ \\
\hline & & & $\downarrow$ \\
\hline 138 & $g-18$ & $g$ - орбиталь & $K^{2} L^{2-6} M^{2-6-10} N^{2-6-10-14} O^{2-6-10-14-18}$ \\
\hline 139 & $f-1$ & $f$ - орбиталь & $K^{2} L^{2-6} M^{2-6-10} N^{2-6-10-14} O^{2-6-10-14-}$ \\
\hline & & & \\
\hline 152 & $f-14$ & $f$ - орбиталь & $K^{2} L^{2-6} M^{2-6-10} N^{2-6-10-14} O^{2-6-10-}$ \\
\hline 153 & $d-1$ & $d$ - орбиталь & $K^{2} L^{2-6} M^{2-6-10} N^{2-6-10-14} O^{2-6-10-14-18} P^{2-6-10-14} Q^{2-6-1} X^{2}$ \\
\hline & & & 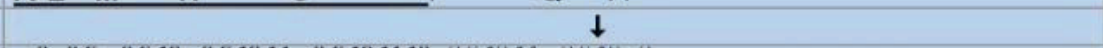 \\
\hline 162 & $d-10$ & d- орбиталь & $K^{2} L^{2-6} M^{2-6-10} N^{2-6-10-14} O^{2-6-10-14}$ \\
\hline 163 & $p-1$ & p- орбиталь & $K^{2} L^{2-6} M^{2-6-10} N^{2-6-1}$ \\
\hline 164 & $p-2$ & p- орбиталь & $K^{2} L^{2-6} M^{2-6-10} N^{2-6-10-14} O^{2-6-10}$ \\
\hline 165 & $p-3$ & p- орбиталь & $K^{2} L^{2-6} M^{2-6-10} N^{2-6-10-14} O^{2-6-10-1}$ \\
\hline 166 & $p-4$ & p- орбиталь & $K^{2} L^{2-6} M^{2-6-10} N^{2-6-10-14} O^{2-6-10-14-18} \mathrm{P}^{2-6}$ \\
\hline 167 & $p-5$ & p- орбиталь & $K^{2} L^{2-6} M^{2-6-10} N^{2-6-10-14} O^{2-6-10-14-18} \mathrm{P}^{2-6-10-14} \mathrm{Q}^{2-6-10} \mathrm{X}^{2-5}$ \\
\hline 168 & $p-6$ & р- орбиталь & $K^{2} L^{2-6} M^{2-6-10} N^{2-6-10-14} O^{2-6-10-14-18} \mathrm{P}^{2-6-}$ \\
\hline \multicolumn{4}{|r|}{ 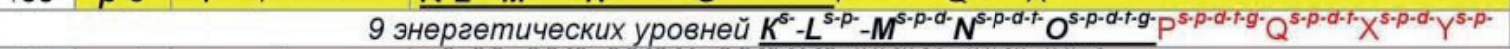 } \\
\hline 169 & $s-1$ & s-орбиталь & $K^{2} L^{2-6} M^{2-6-10} N^{2-6-10-14} O^{2-6-10-14-18} P^{2-6-10-14} Q^{2-6-70} X^{2-6} Y^{7}$ \\
\hline 170 & $s-2$ & s-орбиталь & $K^{2} L^{2-6} M^{2-6-10} N^{2-6-10-14} \mathrm{O}^{2-6-10-14-18} \mathrm{P}^{2-6-}$ \\
\hline 171 & $g-1$ & $g$ - орбиталь & $K^{2} L^{2-6} M^{2-6-10} N^{2-6-10-14} O^{2-6-10-14-18} \mathrm{P}^{2-6-1}$ \\
\hline & & & $\downarrow$ \\
\hline 188 & $g-18$ & g- орбиталь & $K^{2} L^{2-6} M^{2-6-10} N^{2-6-10-14} O^{2-6-10-1}$ \\
\hline 189 & $f-1$ & $f$ - орбиталь & $K^{2} L^{2-6} M^{2-6-10} N^{2-6-10-14} O^{2-6-10-14-18}$ \\
\hline & & & $\downarrow$ \\
\hline 202 & $f-14$ & $f$ - орбиталь & $K^{2} L^{2-6} M^{2-6-10} N^{2-6-10-14} O^{2-6-10-14-18} \mathrm{P}^{2-6-10-14-18} \mathrm{Q}^{2-6-10-14} \mathrm{X}^{2-6} \mathrm{Y}^{2}$ \\
\hline 203 & $d-1$ & $d$ - орбиталь & $K^{2} L^{2-6} M^{2-6-10} N^{2-6-10-14} O^{2-6-10-14-18} \mathrm{P}^{2-6-1}$ \\
\hline & & & \\
\hline 212 & $d-10$ & $d$ - орбиталь & $K^{2} L^{2-6} M^{2-6-10} N^{2-6-10-14} O^{2-6-10-14-18} \mathrm{P}^{2-6-10-}$ \\
\hline 213 & $p-1$ & p- орбиталь & $K^{2} L^{2-6} M^{2-6-10} N^{2-6-10-14} \mathrm{O}^{2-6-10-14-18} \mathrm{P}^{2-6-10}$ \\
\hline 214 & $p-2$ & p- орбиталь & $K^{2} L^{2-6} M^{2-6-10} N^{2-6-10-14} O^{2-6-10-14-18} \mathrm{P}^{2-6-10-14-18} \mathrm{Q}^{2-6-10-14} \mathrm{X}^{2-6-10} \mathrm{Y}^{2-2}$ \\
\hline 215 & $p-3$ & p- орбиталь & $K^{2} L^{2-6} M^{2-6-10} N^{2-6-10-14} O^{2-6-10-14-18} P^{2-6-10-14-18} Q^{2-6-10-14} X^{2-6-10} Y^{2-3}$ \\
\hline 216 & $p-4$ & p- орбиталь & $K^{2} L^{2-6} M^{2-6-10} N^{2-6-10-14} O^{2-6-10-14-18} \mathrm{P}^{2-6-10-14-18} \mathrm{Q}^{2-6-10-14} \mathrm{X}^{2-6-10} \mathrm{Y}^{2-4}$ \\
\hline 217 & $p-5$ & p- орбиталь & $K^{2} L^{2-6} M^{2-6-10} N^{2-6-10-14} O^{2-6-10-14-18} \mathrm{P}^{2-6-10-14-18} \mathrm{Q}^{2-6-10-14} \mathrm{X}^{2-6-10} \mathrm{Y}^{2-5}$ \\
\hline 218 & $p-6$ & p- орбиталь & $K^{2} L^{2-6} M^{2-6-10} N^{2-6-10-14} O^{2-6-10-14-18} \mathrm{P}^{2-6-10-14-18} \mathrm{Q}^{2-6-10-14} \mathrm{X}^{2-6-10} \mathrm{Y}^{2-6}$ \\
\hline
\end{tabular}

К этому же следует отнести понятие о свойствах блочной парности структур, позволяющее на основе электронно-уровневых формул прогнозировать химические элементы за пределами 118-го элемента. В табл. 1 представлены электронно-орбитальные формулы химических элементов для всех блоков, в том числе 5 блока Е, включающего элементы со 119 по 218.

С позиции физики целесообразно рассмотреть результат энергетических взаимодействий, определяющих все свойства вещества. При этом могут быть два подхода, которые определяют две энергетические модели структурного представления вещества. Одна модель с использованием электронно-орбитальных формул (ЭОФ) (табл. 1), другая модель физическая с позиций структурирования на основе квантовых явлений и физических констант (ФК).

Физический метод по сравнению с формальным отличается тем обстоятельством, что он основан на причинно-следственном соответствии энергетических взаимодействий физических полей (причина) пространственно-временного положения электронов оболочек однородных и разнородных химических элементов (следствие). Это положение аналогично представлениям классической механики и может быть определено рядом констант [4].

\section{ЗАКЛЮЧЕНИЕ}

Дальнейшие исследования по формированию структуры вещества в направлении оценки влияния всех видов взаимодействия: сильные и слабые электромагнитные и гравитационные поля, которые будут характеризоваться фундаментальными физическими константами. Авторы продолжат исследования по рассмотрению тех констант, которые влияют на процессы структурообразования и создание новых видов конструкционных материалов. 


\section{REFERENCES}

1. Mendeleev D.I. Osnovy himii [The Fundamentals of Chemistry]. Moscow. Leningrad. Gosudarstvennoe nauchnotekhnicheskoe izd-vo himicheskoj literatury [State scientific and technical publishing house of chemical literature]. In 2 volumes. $13^{\text {th }}$ edition. 1947. V. 1, 624 p. V. 2, 708 p. (In Russian).

2. Gusev B.V., Speransky A.A. Obiomnaya periodicheskaya matrica himicheskih ehlementov [Three-dimensional periodic matrix of chemical elements]. Tekhnika i tekhnologiya silikatov [Technology and engineering of silicates]. Vol. 25, No. 2. 2018, 34-38 p. (In Russian).

3. Gusev B.V., Speransky A.A. et al. Matrichnoe predstavlenie periodichnosti sistemy himicheskih ehlementov [Matrix representation of periodicity of the system of chemical elements]. Russkij inzhener [Russian engineer]. № 4, 52-57 p. (In Russian).

4. Reese. M. Vsego 6 chisel: glavnye sily, formiruyushchie Vselennuyu [Just Six Numbers: The Deep Forces that Shape the Universe]. Transl. from Eng. Moscow, Alpina Ion Fiction, 2018, 226 p. (In Russian).

\section{СПИСОК ЛИТЕРАТУРЫ}

1. Менделеев Д.И. Основы химии. М.-Л.: Государственное научно-техническое изд-во химической литературы. В 2-х томах. 13-е издание. - 1947. - Т. 1 - 624 с.; Т. 2 - 708 с.

2. Гусев Б.В., Сперанский А.А. Объемная периодическая матрица химических элементов. Техника и технология силикатов. - 2018. - Том 25, № 2. - 34-38 с.

3. Гусев Б.В., Сперанский А.А. и др. Матричное представление периодичности системы химических элементов. Русский инженер. - № 4, 52 - 57 с.

4. Рис М. Всего 6 чисел: главные силы, формирующие Вселенную / Мартин Рис, пер. с англ. - М., Альпина ионфикшн. - 2018. -226 с.

\section{INFORMATION ABOUT THE AUTHORS}

Boris V. Gusev, Doctor of technical sciences, Prof., Corresponding Member of the RAS, RAE Acad., head of the Department of Construction Materials and Technologies at Russian University of transport, Moscow, Russia, e-mail: info-rae@mail.ru

Anatoly A. Speransky, PhD in Expertise, Professor, IAE and RAE Academician, Vice President of RAE, Moscow, Russia, e-mail: vibro-vector@yandex.ru

\section{ИНФОРМАЦИЯ ОБ АВТОРАХ}

Гусев Борис Владимирович, д-р техн. наук, проф., член-корр. РАН, акад. РИА, зав. кафедрой «Строительные материалы и технологии» Российского университета транспорта, Москва, Россия, e-mail: info-rae@mail.ru

Сперанский Анатолий Алексеевич, докт. экспертизы, профессор, академик МИА и РИА,

вице-президент РИА, Москва, Россия, e-mail: vibro-vector@yandex.ru 